\title{
Image Enhancement Using Weighted Bi-Histogram Equalization
}

\author{
Thaweesak Trongtirakul \\ Department of Electrical Engineering, \\ Faculty of Industrial Education \\ Rajamangala University of Technology Phra Nakhon \\ 399 Samsen Wachira Phayaban, Dusit, Bangkok Thailand \\ thaweesak.tr@rmutp.ac.th
}

\author{
Nattapong Phanthuna \\ Department of Electrical Engineering, \\ Faculty of Engineering \\ Rajamangala University of Technology Phra Nakhon \\ 1381 Pracharat 1, Bangsue, Bangkok Thailand \\ nattapong100@gmail.com
}

Received: April 18, 2019. Revised: May 29, 2021. Accepted: October 27, 2021. Published: November 17, 2021.

\begin{abstract}
Image enhancement is one of using in various digital signal processing areas. Advances in microcontrollers, microcomputers and computers have developed traditional algorithms in order to improve the quality of the resulting image and have implied many avenues to the design of new innovations using various techniques. This paper proposes contrast enhancement using weighted bi-histogram equalization based on distributed area ratio. Moreover, this technique must use a weighted factor which is calculated by the ratio of the histogram distribution. Likewise, an original image will be equalized by the modification of the probability density function of the gray levels. As result of the experiment, the contrast of resulting image is improved for implement and human perception - as well as also reduces the absolute mean brightness error (AMBE) than the traditional technique of image enhancement.
\end{abstract}

Keywords-Histogram Equalization, Weighted-Histogram Equalization, Area Ratio, Brightness Preservation, Tenengrad, Absolute Mean Brightness Error

\section{INTRODUCTION}

Histogram Equalization (HE) is one of traditional techniques to increase the contrast of the image in digital image processing area. This technique is widely used to improve an image because it is easy to process and is simply. On the other hand, HE technique will produce the resulting image that is over-enhancement (also known as the quantum jump [1]). For instance, if an original image consists of most dark pixels, the resulting image will be extremely washed-out as shown in Fig.1. If an original one consists of most light pixels, the unwanted information will be cut-in as shown in Fig.2. In two cases, the resulting image will absolutely lost important information during histogram distribution.

Accordingly, there are many research [2], [3], [4] that present to preserve the brightness error. Thus, this proposed method aims to reduce its error using the weighted bihistogram equalization which deals with problems.

\section{HISTOGRAM EQUALIZATION}

Define $\mathbf{X}=\{X(i, j)\}$ is an original image of discrete gray levels as $\left\{X_{0}, X_{1}, \ldots, X_{L-1}\right\}$, where $X(i, j)$ is an original image intensity of the pixels $(i, j)$ and $X(i, j) \in\left\{X_{0}, X_{1}, \ldots, X_{L-1}\right\}$.
Step 1: calculate the probability density function (PDF) is determined

$$
p(l)=\frac{n_{l}}{N} \quad ; l=0,1, \ldots, L-1
$$

where $n_{l}$ is the $l$-th of the gray level in the original image $\mathbf{X}$ and $N$ is the total number of pixel in the original image.

Step 2: calculate the cumulative density function (CDF) is determined as

$$
c(l)=\sum_{i=1}^{L-1} p(l)
$$

Note $0 \leq c(l) \leq 1$

Step 3: calculate the new gray level of the resulting image using the mapping function is determined as

$$
f(l)=X_{0}+\left(X_{L-1}-X_{0}\right) \cdot c(l)
$$

where $X_{0}$ is the starting gray level of the resulting image. $X_{L-1}$ is the ending gray level of the resulting image. Typically, $X_{0}$ and $X_{L-1}$ are defined as 0 and 255 , respectively.
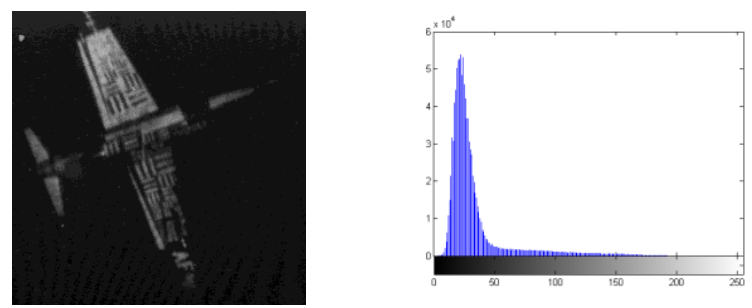

(a) 

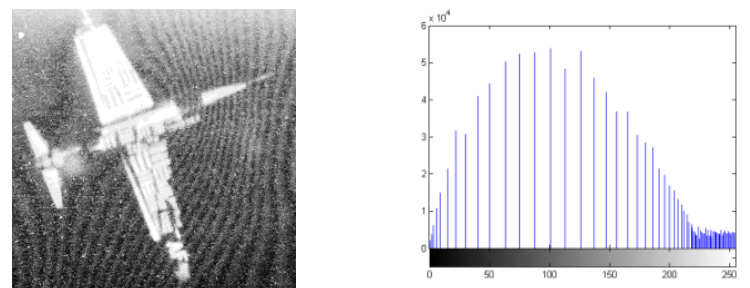

(b)

Fig. 1 image that consists of most dark pixels (a) original image and its corresponding histogram (b) resulting image using $\mathrm{HE}$ and its corresponding histogram

Step 4: represent the new gray level from step 3 into the original image. Then, the resulting image can be defined as

$$
\mathbf{Y}=\{f(X(i, j) \mid \forall x(i, j) \in \mathbf{X})\}
$$

Typically, the histogram Equalization starting from $X_{0}=0$ to $X_{L-1}=255$ is widely known as "Global Histogram Equalization (GHE)".

HE algorithm always makes the high contrast of a resulting image to emphasise some information in an image however it also affects brightness errors in resulting one. [5]

\section{BRIGHTNESS PRESERVATION USING WEIGHTED BI-Histogram EQUALIZATION}

Define $\mathbf{X}_{\text {ori }}=\left\{X_{\text {ori }}(i, j)\right\}$ is an original image of discrete gray level as $\left\{X_{0}, X_{1}, \ldots, X_{L-1}\right\}$, where $X_{o r i}(i, j)$ is an intensity of the pixel $(i, j)$ and $X_{\text {ori }}(i, j)$ is the number of $\left\{X_{0}, X_{1}, \ldots, X_{L-1}\right\}$

Step 1: Calculate the PDF is determined as

$$
p(a)=\frac{n_{a}}{N} ; a=0,1, \ldots, L-1
$$

where $a$ is the a-th of the gray level in the image $\mathbf{X}$ and $n$ is the total number in each bin of the gray level. $N$ is the total number of the whole image.
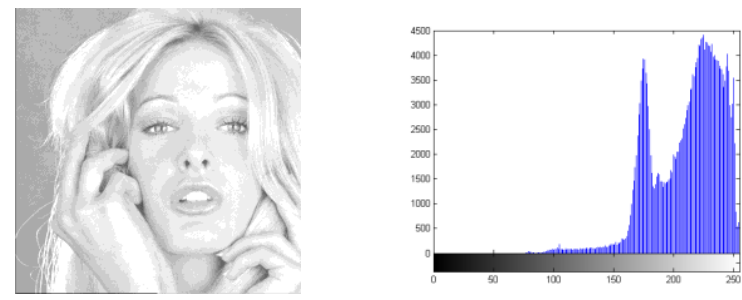

(a)

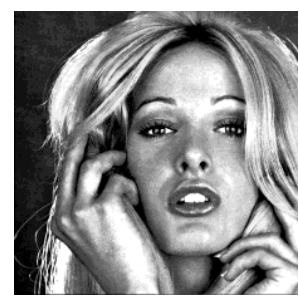

(b)

Fig. 2 image that consists of most light pixels

(a) original image and its corresponding histogram

(b) resulting image using $\mathrm{HE}$ and its corresponding histogram

Step 2: Calculate the CDF is determined as

$$
c(a)=\sum_{a=1}^{L-1} p(a)
$$

Step 3: Determine the threshold level for dividing the whole histogram of the original image into two sections is determined as

$$
\tau=\lim _{c \rightarrow 0.5} f(X)
$$

the -th gray level is used for dividing the whole histogram of the original image into two sections whereas about 50-percent of pixels are originated from the first region - as well as remaining pixels are from the second region.

$\tau$

Step 4: Calculate weighting factors are determined as

$$
\begin{gathered}
w_{1}=\frac{s_{0}}{2 \tau} \\
w_{2}=\frac{s_{1}-\frac{s_{1}}{2}}{256}+\frac{1}{2}
\end{gathered}
$$

where is the starting gray level of the original histogram. $s_{1}$ is the ending gray level of the original histogram as shown in Fig.3

Define $s \mathbf{X}_{1}=\left\{x_{1}(i, j)\right\}$ is the first region of the original histogram started from $L$ discrete gray level as $\left\{s_{0}, s_{0+1}, \ldots, \tau\right\} . \mathbf{X}_{2}=\left\{x_{2}(i, j)\right\}$ is the second region of the original histogram started from $L$ discrete gray level as $\{\tau+1, \tau+2, \ldots, L-1\}$. 


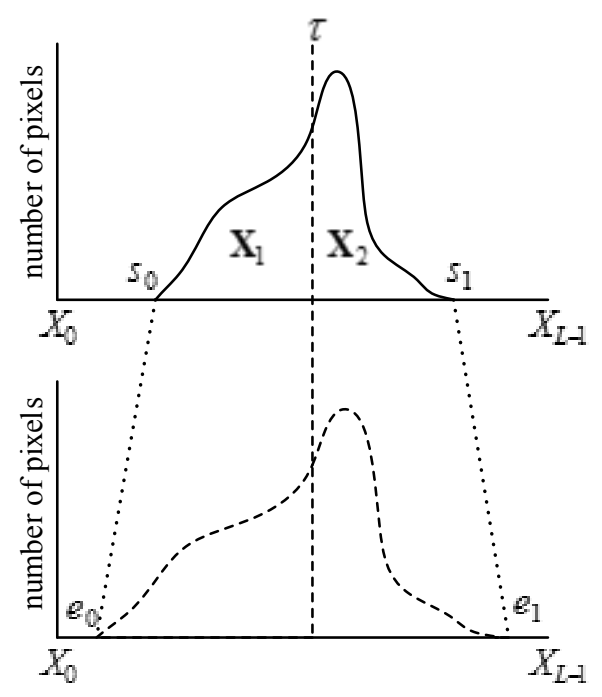

Fig.3 The concept of our proposed method

Step 5: Calculate PDF in each region is determined as

$$
\begin{gathered}
p_{1}(y)=\frac{n_{y}}{N_{1}} ; y=0,1, \ldots, \tau \\
p_{2}(z)=\frac{n_{z}}{N_{2}} ; z=\tau+1, \tau+2, \ldots, L-1
\end{gathered}
$$

where $n_{y}$ is the $y$-th of the gray level in the image $\mathbf{X}_{1}$ started from -th gray level to the -th gray level . $N_{1}$ is the total number of pixels in the image $\mathbf{X}_{1} \cdot n_{z}$ is the $z$-th of the gray level in the image $\mathbf{X}_{2}$ started from the +1-th gray level to the $L-1$-th gray level. $N_{2}$ is the total number of pixels in the image ${ }^{s} \mathbf{X}_{2}$.

Step 6: Calculate CDF in each region is ${ }^{\tau}$ determined as

$$
\begin{aligned}
& c_{1}(y)=\sum_{y=0}^{\tau} p_{1}(y) \\
& c_{2}(z)=\sum_{z=\tau+1}^{L-1} p_{2}(z)
\end{aligned}
$$

Note $0 \leq c_{1}(y) \leq 1$ and $0 \leq c_{2}(y) \leq 1$ as well.

Step 7: Calculate the mapping function from step 13 in order to represent the new gray level of the resulting image

$$
\begin{gathered}
f(y)=\tau\left[w_{1}+c(y) \cdot\left(1-w_{1}\right)\right] \\
f(z)=(\tau+1)+\left[X_{L-1} \cdot w_{2}-(\tau+1)\right] c_{2}(z)
\end{gathered}
$$

Step 8: represent the new gray level of the resulting image from step 7 describes as

$$
\begin{gathered}
\mathbf{Y}=\mathbf{Y}_{1} \cup \mathbf{Y}_{2} \\
\mathbf{Y}=Y_{1}(f(y)) \cup Y_{2}(f(z))
\end{gathered}
$$

The resulting image is equalized independently whereas $\mathbf{Y}$ is a resulting image. $\mathbf{Y}_{1}$ is the resulting sub-image of sub-histogram $\mathbf{X}_{1}$ which is equalized by $f(y)$ and $\mathbf{Y}_{2}$ is the resulting sub-image of sub-histogram $\mathbf{X}_{2}$.

Table 1 Absolute Mean Brightness Error (AMBE) comparison with traditional methods and our proposed method (WBHE)

\begin{tabular}{|l|r|r|r|}
\hline Image & \multicolumn{1}{|c|}{ GHE } & \multicolumn{1}{c|}{ BBHE } & \multicolumn{1}{|c|}{ WBHE } \\
\hline Airport & 45.96031 & 17.21593 & 15.43191 \\
Car and APCs & 13.93107 & 11.38658 & 9.63023 \\
Chemical Plant & 23.90703 & 12.16783 & 2.30620 \\
Girl & 17.37088 & 12.16783 & 0.06348 \\
Moon Surface & 1.25349 & 1.81264 & 1.32314 \\
Splash & 25.44266 & 11.56768 & 0.41983 \\
Tank & 21.76308 & 12.75653 & 5.79171 \\
Tiffany & 82.16163 & 38.03572 & 37.81729 \\
Track and APCs & 22.63727 & 11.74712 & 0.22270 \\
Truck & 23.03217 & 14.91651 & 7.54406 \\
U2 & 98.81740 & 41.47091 & 25.85977 \\
\hline
\end{tabular}

\section{CONCLUSION}

Our proposed method is presented to overcome both GHE and BBHE that existing method. Our proposed method is able to preserve the resulting images with high contrast enhancement as shown in Fig.4 and Fig. 5. Moreover it also protects against AMBE simultaneously as shown in Table 1.

\section{ACKNOWLEDGMENT}

The authors are grateful to the Faculty of Engineering, Rajamangala University of Technology Phra Nakhon (RMUTP) and Ministry of Science and Technology, for the support in conducting this study. Thanks friends for the helpful exchange of information.

\section{REFERENCES}

[1] Se-Hwan Yun, Jin Heon Kim and Suki Kim, Contrast Enhancement using a Weighted Histogram Equalization, 2011 IEEE International Conference on Consumer Electronics (ICCE), 2011, pp. 203 - 204.

[2] Yeong-Taeg Kim, Contrast Enhancement Using Brightness Preserving BiHistogram Equalization, IEEE Transactions on Consumer Electronics, Vol. 43, No. 1 February 1997, pp. $1-8$

[3] R. C. Gonzalez and P. Wints, Digital Image Processing, $2^{\text {nd }}$ Edition, Addison-Wesley Publishing Co., Reading, Massachusetts, 1987.

[4] OGE Marques, Practical Image and Video Processing Using MATLAB, A John Wiley and Sons. Inc. Publication, 2011.

[5] Soong-Der Chen, Ramli and A.R., Minimum Mean Brightness Error BiHistogram Equalization in Contrast Enhancement, 2003 IEEE Transections on Consumer Electronics, Vol. 49, Issue 4, pp. 1310 - 1319.

\section{Creative Commons Attribution License 4.0 (Attribution 4.0 International, CC BY 4.0)}

This article is published under the terms of the Creative Commons Attribution License 4.0

https://creativecommons.org/licenses/by/4.0/deed.en_US 

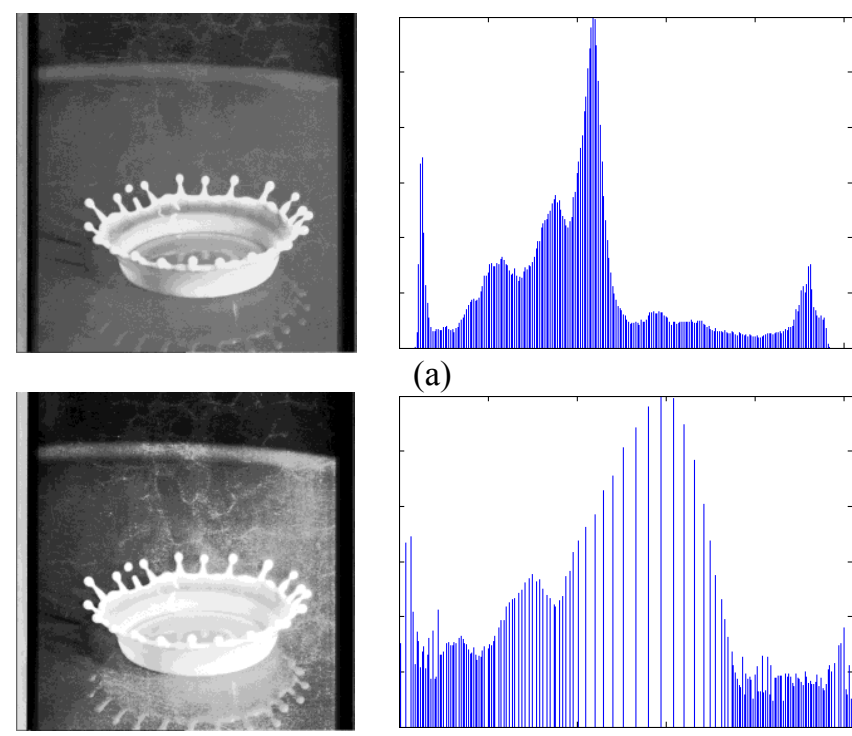

(a)

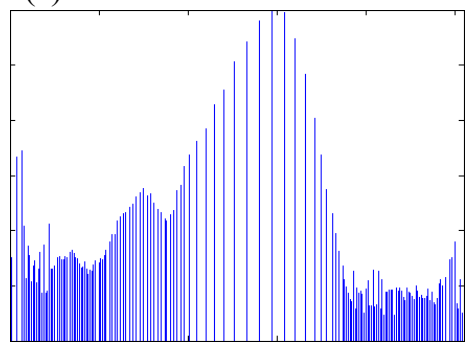

(c)
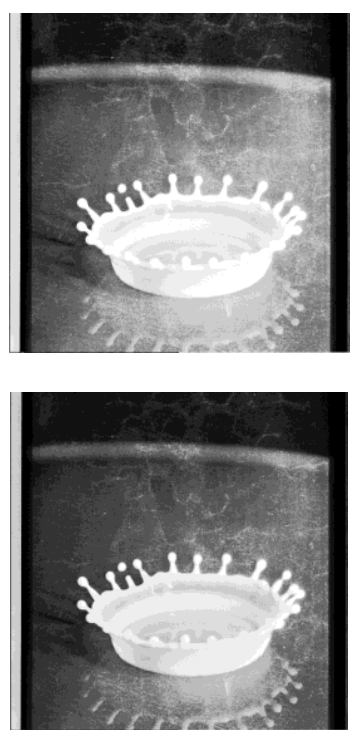

(b)

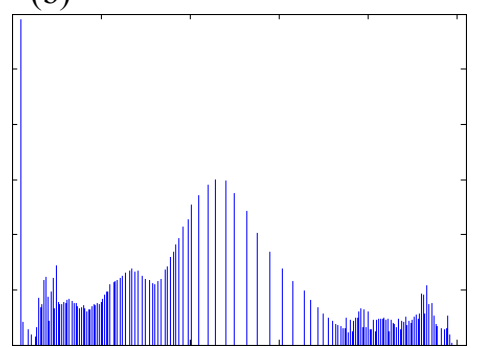

(d)

Fig. 4 Splash Images and its corresponding histograms comparison

(a) Original image (b) resulting image of (a) using GHE

(c) resulting image of (a) using BBHE (d) resulting image of (a) using WBHE and its histogram
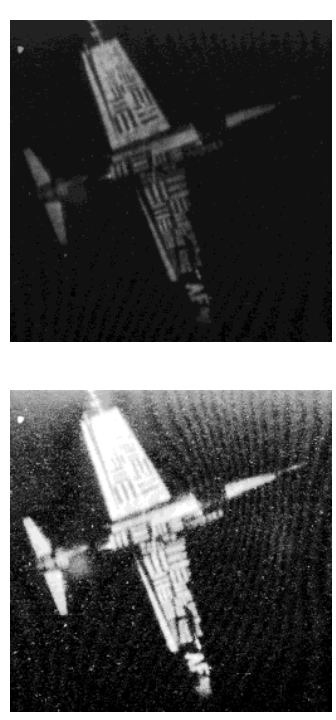
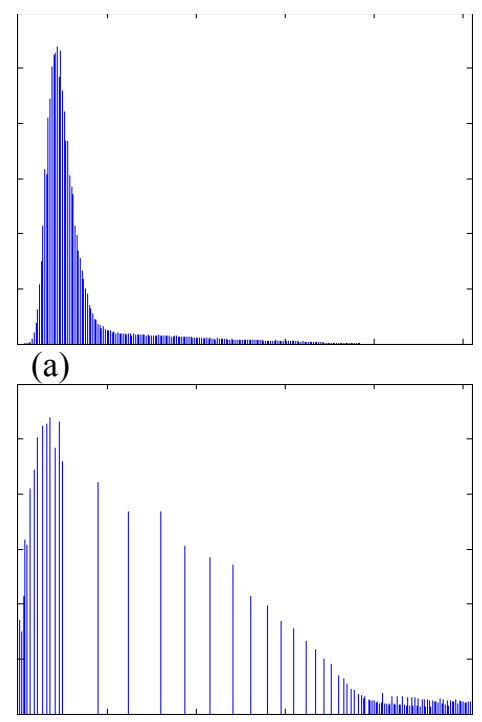

(c)
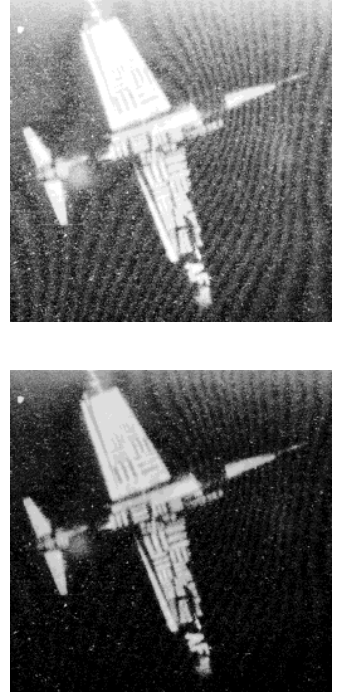

(d)

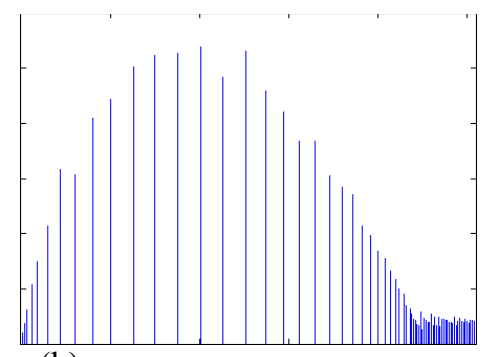

(b)

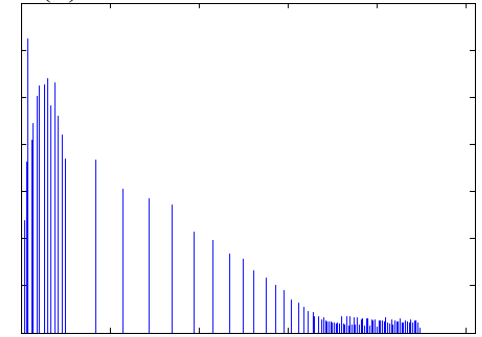

Fig. 5 U2 Images and corresponding histograms comparison

(a) Original image and its corresponding histogram (b) resulting image of (a) using GHE

(c) resulting image of (a) using BBHE (d) resulting image of (a) using WBHE 